\title{
Beamforming Scheme for MIMO Relay Based 5G and beyond Wireless Network
}

\author{
Ali Mahamoud Ali', Bakhit Amine Adoum,2, Idriss Saleh Bachar², Mahamoud Youssouf Khayal' \\ ${ }^{1}$ Laboratoire de Mathématiques, Modélisation, Informatique, Applications et Simulation, LARTIC, FSEA/École Doctorale Sciences \\ Techniques et Environnement, CNRD, Université de N'Djamena, N’Djamena, Tchad \\ ${ }^{2}$ Ecole Nationale Superieure des Technologies de l'Information et de la Communication (ENASTIC), N'Djamena, Tchad \\ Email: alimahamoud890@yahoo.fr, bakhitamine@yahoo.fr, idrissay@hotmail.com,mykhayal@yahoo.fr
}

How to cite this paper: Ali, A.M., Adoum, B.A., Bachar, I.S. and Khayal, M.Y. (2021) Beamforming Scheme for MIMO Relay Based $5 \mathrm{G}$ and beyond Wireless Network. Open Journal of Applied Sciences, 11, 1157-1176. https://doi.org/10.4236/ojapps.2021.1110087

Received: September 22, 2021

Accepted: October 26, 2021

Published: October 29, 2021

Copyright $\odot 2021$ by author(s) and Scientific Research Publishing Inc. This work is licensed under the Creative Commons Attribution International License (CC BY 4.0).

http://creativecommons.org/licenses/by/4.0/ (c) (i) Open Access

\begin{abstract}
In this paper, in order to attain the maximum ergodic capacity and significantly increase the spectral efficiency of wireless communication systems, novel linear beamforming is proposed for dual-hop amplify-and-forward (AF) multi-relay networks. The linear beamforming is designed based on the maximization of the signal-to-interference-plus-noise ratio (SINR) and signal-toleakage-and-noise ratio (SLNR). The channel state information (CSI) is used in applying this new design to multi-relay (MR) nodes between the source and relays as well as relays and destination. The beamforming optimization problem is solved by using the Fukunaga-Koontz Transform (FKT). The scheme can achieve intra-node array and distributed gains by using multiple antennas and multi-relays (MRs). The performance of the proposed scheme demonstrates that by considering interference mitigation criteria the ergodic capacity at a significant level is improved as compared to the conventional techniques. Therefore, the proposed techniques based on the maximization of the signal-to-interference-plus-noise ratio (SINR) and signal-to-leakage-and-noise ratio (SLNR) relay processing outperform other conventional techniques in case of a multi-relay dual-hop network in terms of ergodic.
\end{abstract}

\section{Keywords}

Beamforming, Ergodic Capacity, MIMO, Multi-Relay Network

\section{Introduction}

Due to high data rate, delivery dream, and interference, free demand of wireless services, the existing and future mobile communication technologies such as $4 \mathrm{G}$ and 5G, and cognitive radio are employing advanced technologies like MIMO 
and multi-relay (MR) systems. The technologies are being pursued to attend reliable communications with high data rate and low energy consumption providing high service quality. In wireless networks, multiple MIMO relays are used to achieve diversity in signal transmission and to improve overall network performance confrontations. Due to path loss, shadowing, multipath fading and signal interference effects, the channel capacity, reliability, and quality are debased in mobile communication. This is assumed as common transmission impairments of wireless transmission. To tackles these issues, micro-base stations are deployed which require substantial running and capital costs. For the future as well as existing wireless communication systems, a reliable and cost-effective technique is used called the relay-assisted transmission technique. This technique can enhance the quality of the wireless network between the source and destination and can improve base station coverage at the cell edge [1]. Relay-assisted communications have become popular after the great work discussed in [2]. Due to the profitable assistance of MIMO relay nodes to the MIMO systems, the existing and future wireless networks, LTE, and advanced networks [3] are widely considering the relay-assisted MIMO (RA-MIMO) technologies as a vibrant candidate to support high data demands [4].

The amplify-and-forward (AF) relay technology is more attractive because of low complexity and simplicity of application and decode-and-forward (DF) relay in which processing delay occurs due to the decoding and encoding process [5]. Beamforming can also enhance the performance of relay-based networks. Recently, many beamforming techniques have been proposed to enhance the performance of relay based networks and as a key enabling technology for 5G communication [6]-[19]. An appropriate design of beamforming for AF relay can efficiently reduce the level of interference at the receiving and transmitting sides, which helps to obtain the optimal capacity. Adequate researches are performed for beamforming design that can approach the upper-bound capacity derived in [11] for MR-DH-MIMO system. The capacity scaling of a RA-MIMO network is discussed and it is proved that the capacity of the MR-MIMO network escalates by increasing the relay nodes in the cluster [11]. The ergodic capacity of a MR network is also analyzed by considering a single antenna at the relay nodes [12]. A multi-relay wireless system is studied, which proposes the $\mathrm{QR}$ decomposition (QRD) based relay receive and transmit beamforming to achieve better network performance [13]. It is also claimed that multiple uses of QRD operation at transceiver of MR nodes can increase the cost of QRD operation [14]. The authors have also proposed an efficient algorithm in [14] for the MR network which employs the same number of antennas to each node. A matched filter (MF) is used in this design as the relay receive beamforming whereas, for the relay transmit beamforming, regularized zero-forcing (RZF) is used with the objectives to achieve intra-array and distributed gain. The performance of the MF-RZF is better as compared to the techniques discussed in [13]. In this design, the RZF reaches the zero-forcing ( $\mathrm{ZF})$ scheme, if the regularization parameter value becomes very small or near to zero, causing noise enhancement. However, a high 
regularization parameter value causes the equivalent channel matrix to deviate from the diagonal matrix, causing interference and extra power expenditure throughout data streams [15] [16] [17]. Since, a specific amount of interference is allowed in the RZF technique [18], therefore with a non-zero regularization parameter value, the interference cannot be canceled completely [19].

In noise-limited scenarios, the MF beamforming technique is important to deliver better performance. However, the MF performance in interference-limited scenarios is poor [20]. Therefore, for both the scenarios, it is necessary to have the techniques that perform efficiently, in practical communication systems, cochannel interference as well as adjacent-channel interference is present [21], which should be included in the objective function of designing the beamforming for MRs. The designs based on the signal-to-interference-plus-noise ratio (SINR) maximization (SINR-Max) and signal-to-leakage-and-noise ratio (SLNR) maximization (SLNR-Max) criteria perhaps are the capable contender to overcome this problem. The techniques based on SINR-Max consider noise and interference and make the system perform better than the techniques used in [14] and [22] for relay receive beamforming. Nevertheless, the beamforming computational load has usually a large value, and to lower that value, efficient Fukunaga-Koontz Transform (FKT) can be used to design the relay receive beamforming based on SINR-Max [23] [24]. For MRs in a DH network, SLNR-Max is used which is based on downlink precoding [22].

However, this paper contributes to the design of relay receive beamforming based on SINR-Max using the FKT. It is worth mentioning that the scheme handles the noise and interference in both, the MR receives beamforming design and downlink precoding design. This novel relay processing design can achieve the distributed and intra-node array gain by controlling the interference at the reception of the relay nodes and intra-user interference because of the leakage at the destination. Intra-node array gain is achieved when multiple antennas are deployed at individual node of the network, whereas, the distributed array gain is obtained through various relay nodes connected in parallel [14]. The novelty of relaying scheme is in SINR-Max and SLNR-Max beamforming designs, which uses FKT for multiple AF relay nodes to obtain these types of gains. The proposed scheme can improve the enactment terms of ergodic capacity. It can make it possible to control interference among various antennas of the relays and at the destination node.

\section{System Model}

In this research, a dual-hop single user AF MR-based MIMO (DH-AF-MIMO) network is studied as presented in Figure 1. It consists of the transmit antennas

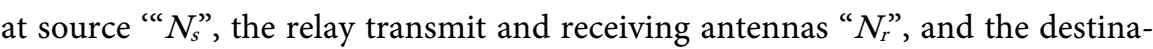
tion antennas " $N_{d}$ ". Signal vector " $\mathcal{S}$ " is aired to AF relay nodes by the source. The transmission between the source and destination is not considered because of long-distance deep fading. The MR nodes were joined in parallel to help to 


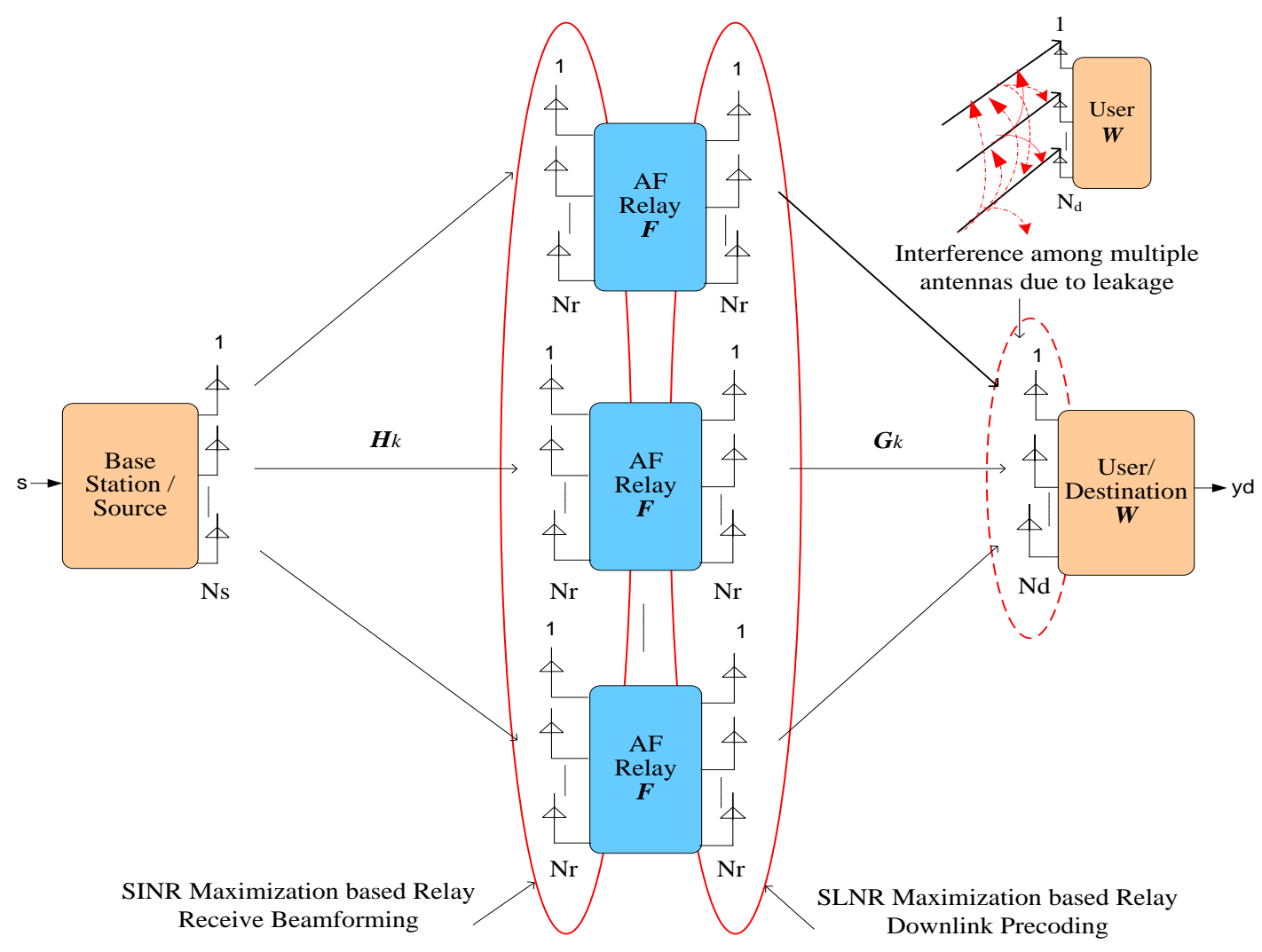

Figure 1. DH-AF-MIMO.

establish the communication between the source and destination. The source node first sends the information signal, processes it, and then forwards it to the destination by using MR nodes, which possesses multiple antennas at the individual relay. The relays with multiple antennas give better performance [25]. The mode of transmission of relay nodes is supposed to be the half-duplex mode. At the relay nodes, the channel knowledge of both channels. The channels are supposed to be impartial and uniformly distributed, experiencing the same Rayleigh flat fading effect.

The information signal transmission is distributed into two phases. In the first phase, the modulated signal vector " $s$ " is transmitted to MR nodes by the source as $N_{s} \times 1$ and it satisfies the source power constraint as:

$$
E\left\{\boldsymbol{s s}^{H}\right\}=\frac{P_{s}}{N_{s}} \boldsymbol{I}_{N_{s}}
$$

where;

$P_{s}=$ source transmit power

$\boldsymbol{I}_{N_{\mathrm{s}}}=$ identity matrix of $N_{s} \times N_{s}$.

The information signal at the $k^{\text {th }}$ relay node is as follows:

$$
\boldsymbol{s}_{r k}=\sum_{k=1}^{K} \boldsymbol{H}_{k} \boldsymbol{s}+\boldsymbol{n}_{r k}
$$

where,

$$
\boldsymbol{H}_{k}=\left[\boldsymbol{H}_{1}^{\mathrm{T}}, \boldsymbol{H}_{2}^{\mathrm{T}}, \cdots, \boldsymbol{H}_{K}^{\mathrm{T}}\right]^{\mathrm{T}} \text {, which is the channel matrix of dimension }
$$


“ $N_{r} K \times N_{s}$;

$$
\begin{gathered}
k=1,2, \cdots, K . \\
E\left\{\boldsymbol{n}_{r k} \boldsymbol{n}_{r k}^{H}\right\}=\sigma_{r}^{2} \boldsymbol{I}_{N_{r}}
\end{gathered}
$$

where;

$$
\sigma_{r}^{2}=\text { noise variance at the relay node. }
$$

In the second stage, linear processing is performed through the AF relay nodes by the multiplication of a beamforming matrix " $\boldsymbol{F}_{k}$ " of " $N_{r} \times N_{r}$ " dimension with the received signal vector " $\boldsymbol{s}_{r, k}$ " and then forwarded to the destination. The network performance is improved by the beamforming matrix and the relay transmit power constraint is satisfied as:

$$
E\left\{\boldsymbol{r}_{k}^{H} \boldsymbol{r}_{k}\right\} \leq P_{k}
$$

where;

$$
P_{k}=\text { relay transmit power }
$$

The transmitted signal by $k^{\text {th }}$ relays to the destination is shown as:

$$
\boldsymbol{r}_{k}=\sum_{k=1}^{K} \boldsymbol{F}_{k} \boldsymbol{H}_{k} \boldsymbol{s}+\boldsymbol{n}_{r k}
$$

where;

$\boldsymbol{r}_{k}=k^{\text {th }}$ relay node transmit power

Therefore, the condition of a power constraint for the precoded signal that is transmitted by AF relay terminals is given as:

$$
\delta\left(r_{k}\right)=\operatorname{tr}\left\{\boldsymbol{F}_{k}\left(\frac{P_{s}}{N_{s}} \boldsymbol{H}_{k} \boldsymbol{H}_{k}^{H}+\sigma_{r k}^{2} \boldsymbol{I}_{N_{r}}\right) \boldsymbol{F}_{k}^{H}\right\} \leq P_{k}
$$

where;

$\rho_{k}=$ power normalizing coefficient [26]

This can also regulate the relay transmit power which is given as:

$$
\rho_{k}=\sqrt{\frac{P_{k}}{\delta\left(r_{k}\right)}} .
$$

After relay linear processing, the received signal is shown as:

$$
\boldsymbol{y}=\sum_{k=1}^{K} \rho\left(r_{k}\right) \boldsymbol{G}_{k} \boldsymbol{F}_{k} \boldsymbol{H}_{k} \boldsymbol{s}+\sum_{k=1}^{K} \boldsymbol{G}_{k} \boldsymbol{F}_{k} \boldsymbol{n}_{r k}+\boldsymbol{n}_{d}
$$

where;

$\boldsymbol{G}_{k}=$ complex channel matrix of " $N_{s} \times N_{r} K$ " dimension between the $k^{\text {th }}$ relay nodes $k=1,2, \cdots, K$ and the destination i.e. $\boldsymbol{G}_{k} \in \mathbb{C}^{N_{d} \times N_{r}}$ channels where $\boldsymbol{G}_{k}=\left[\boldsymbol{G}_{1}, \boldsymbol{G}_{2}, \cdots, \boldsymbol{G}_{k}\right]$.

The elements of $\boldsymbol{g}_{j, i}$ symbolize the channel gain from the relay transmit antenna " $\vec{i}$ " to the destination receive antenna " $\vec{j}$ ' i.e. $i=1, \cdots, N_{r}$ and $j=1, \cdots, N_{d}$. Since, $\boldsymbol{n}_{d}$ is $\boldsymbol{n}_{d} \in \mathbb{C}^{N_{d}}$ CGN with zero-mean and covariance, which meets the noise variance as:

$$
E\left\{\boldsymbol{n}_{d} \boldsymbol{n}_{d}^{H}\right\}=\sigma_{d}^{2} \boldsymbol{I}_{N_{d}}
$$

where, 
$\sigma_{d}^{2}=$ noise power at the destination.

\section{Linear Beamforming for Multi-Relay Nodes}

In relay receive beamforming, the cancellation of interference by the ZF technique will cost noise enhancement. Nevertheless, the SNR is maximized by minimizing the noise with the help of the MF beamforming technique but it does not take into consideration the interference cancellation. To deal with this issue, the SINR-Max criterion based on the relay receive beamforming is designed using the FKT. Therefore, the criterion like SINR-Max can provide a reconciliation between the noise and interference by eliminating the interference and minimizing the noise [27]. The issue of interference is faced because of the leakage signal from the desired signals that are forwarded by MR nodes at the designation. To deal with this issue of optimization, the SLNR-Max-based linear precoding technique is designed for MR downlink using the FKT as discussed in [24]. The relay processing technique based on SINR-Max and SLNR-Max for multiple AF relay nodes is proposed as an alternate technique to the conventional relay processing schemes.

\subsection{SINR Maximization Based Beamforming for $K$ Relays}

In this section, SINR-Max based relay receive beamforming for multiple AF relays is designed and discussed using the FKT. For relay receivers, the beamforming vectors can be used in such a way that at $k$ relay nodes, the beamforming can maximize the SINR. The interference is controlled by the beamforming between multiple antennas of MRs for the network as seen in Figure 1. " $\boldsymbol{H}_{k}$ " represents the channel between the source and MR nodes i.e. $\boldsymbol{H}_{k} \in C^{N_{k} \times N_{s}}$. The channel matrix for $K$ relay nodes is given as:

$$
\boldsymbol{H}_{c}=\left[\boldsymbol{H}_{1}^{H}, \boldsymbol{H}_{2}^{H}, \cdots, \boldsymbol{H}_{K}^{H}\right]
$$

where; $\boldsymbol{H}_{c}$ represents the combined matrix.

For each relay receive antenna, the channel matrix is given as:

$$
\hat{\boldsymbol{H}}_{k}^{l}=\left[\hat{\boldsymbol{H}}_{k}^{l^{H}}, \boldsymbol{H}_{k-1}^{H}, \boldsymbol{H}_{k+1}^{H}, \cdots, \boldsymbol{H}_{K}^{H}\right]^{H}
$$

In an MR system, the SINR, represented by “ $\gamma_{k}^{l}$ ”, is obtained when the received signal power is divided by the indesired signal and noise power at $k$ relays. It is expressed as:

$$
\gamma_{k}^{l}=\frac{\left\|\boldsymbol{h}_{k}^{l} \boldsymbol{b}_{k}^{l}\right\|_{F}^{2}}{\sigma_{r}^{l^{2}}+\sum_{\substack{l=1 \\ l \neq k}}^{L}\left\|\boldsymbol{H}_{i} \boldsymbol{b}_{k}^{l}\right\|_{F}^{2}+\sum_{\substack{j=1 \\ j \neq k}}^{N_{k}}\left\|\boldsymbol{h}_{i} \boldsymbol{b}_{k}^{l}\right\|_{F}^{2}}
$$

It can also be written as:

$$
\gamma_{k}^{l}=\frac{\boldsymbol{b}_{k}^{l^{H}}\left(\boldsymbol{h}_{k}^{l^{H}} \boldsymbol{h}_{k}^{l}\right) \boldsymbol{b}_{k}^{l}}{\boldsymbol{B}_{k}^{H^{H}} \boldsymbol{H}_{k}^{l^{H}} \boldsymbol{H}_{k}^{l} \boldsymbol{B}_{k}^{l}+\sigma_{r k}^{l^{2}} \boldsymbol{I}_{N_{r}}}
$$

where, 
$\boldsymbol{b}_{k}^{l}=$ beamforming vector

$\boldsymbol{h}_{k}^{l}=$ complex row vector of the $I^{\text {th }}$ receive antennas of $k$ relay nodes i.e.

$l=1, \cdots, N_{k}$

$\sigma_{r k}^{l^{2}}=$ noise variance at $I^{\text {h }}$ antenna elements for $k^{\text {th }}$ relays i.e.

$\sigma_{r k}^{l^{2}}=\sigma_{1}^{l^{2}}, \cdots, \sigma_{K}^{l^{2}}$ and $\boldsymbol{b}_{k}^{l}=\boldsymbol{b}_{1}, \cdots, \boldsymbol{b}_{N_{k}}$, whichis vector-by-vector relay beamforming for an individual antenna element of the $k^{\text {th }}$ relays.

The numerator term in (9) is representing the desired signal, while the term in the denominator is representing the combination of interference and noise power at the $I^{\mathrm{h}}$ receive antennas of $k^{\text {th }}$ relays. The vectors are developed independently, based on the vector-by-vector relay beamforming. Therefore, the antenna-wise SINR-Max beamforming at " $k$ " relays is set as:

$$
\hat{\boldsymbol{B}}_{k}^{l}=\arg \max _{\boldsymbol{B}_{k}^{l} \in C^{N_{r} \times 1}} \operatorname{SINR}_{k}^{l}\left(\boldsymbol{B}_{k}^{l}\right) .
$$

Or

$$
\begin{aligned}
\boldsymbol{b}_{k}^{l}=\arg \max _{\boldsymbol{b}_{k}^{l} \in C^{N_{r} x}} \frac{\boldsymbol{b}_{k}^{l^{H}}\left(\boldsymbol{h}_{k}^{l^{H}} \boldsymbol{h}_{k}^{l}\right) \boldsymbol{b}_{k}^{l}}{\boldsymbol{B}_{k}^{l^{H}} \boldsymbol{H}_{k}^{l^{H}} \boldsymbol{H}_{k}^{j} \boldsymbol{B}_{k}^{l}+\sigma_{r k}^{l^{2}} \boldsymbol{I}_{N_{r}}} . \\
\boldsymbol{B}_{k}=\left[\boldsymbol{b}_{k}^{1}, \cdots, \boldsymbol{b}_{k}^{N_{k}}\right] .
\end{aligned}
$$

where;

$$
\begin{aligned}
& \boldsymbol{B}_{k}=\text { beamforming matrix for } k \text { relays } \\
& \qquad \begin{array}{r}
k=1, \cdots, K \\
l=1, \cdots, N_{k}
\end{array}
\end{aligned}
$$

\subsection{SLNR Based MR Downlink Precoding Design}

As the interference caused by the signal leakage cannot be canceled by using conventional techniques like the RZF technique, which uses an adjustable parameter to control the interference. However, the interference is not canceled out completely by the non-zero value of the regularization parameter. Although, the leakage-based downlink precoding can show robustness against the interference between multiple antennas at the destination. In this design, the interference due to leakage and noise at the destination side is taken into account for vector by vector downlink precoder design as discussed in [23] and [24]. The proposed technique reduces the interference resulted caused by the signal leakage from the wanted signal among multiple antennas. Figure 1 illustrates the concept of the leakage signals from the desired signals, due to which interference is caused by other antennas. This design aims to reduce the interference that can help in improving the network ergodic capacity.

The channel matrix between the destination and MR nodes at the downlink of the given system is represented by the notation " $G_{k}$ ".

$$
\boldsymbol{G}_{k}=\left[\boldsymbol{G}_{1}^{H}, \boldsymbol{G}_{2}^{H}, \cdots, \boldsymbol{G}_{K}^{H}\right]^{H}
$$

For $f^{\text {th }}$ antennas of $k$ relay nodes to the destination, the channel matrix is given as: 


$$
\boldsymbol{G}_{k}^{j}=\left[\boldsymbol{G}_{k-1}^{H}, \boldsymbol{G}_{k+1}^{H}, \cdots, \boldsymbol{G}_{K}^{H}\right]^{H}
$$

The relay precoder design is developed with the relay power constraint as:

$$
\begin{gathered}
\hat{\boldsymbol{F}}_{k}^{j}=\arg \max _{\boldsymbol{F}_{k}^{j} \in C^{N_{r} \times 1}} \operatorname{SLNR}_{k}^{j}\left(\boldsymbol{F}_{k}^{j}\right) . \\
\text { s.t } \delta\left(r_{k}\right) \leq P_{r} .
\end{gathered}
$$

where, “ $\boldsymbol{F}_{k}^{j}$ ” is the $k^{\text {th }}$ RTP for $f^{\text {th }}$ receiving antennas.

The SLNR per antenna is obtained when the desired signal power is divided by the leakage signal and noise power and is given by:

$$
P A-S L N R_{k}^{j}=\frac{\left\|\boldsymbol{g}_{k}^{j} \boldsymbol{f}_{k}^{j}\right\|_{F}^{2}}{\sum_{\substack{i=1 \\ j \neq k}}^{L}\left\|\boldsymbol{G}_{k} \boldsymbol{f}_{k}^{j}\right\|_{F}^{2}+\sum_{\substack{i=1 \\ j \neq k}}^{N_{d}}\left\|\boldsymbol{g}_{k} \boldsymbol{f}_{k}^{j}\right\|_{F}^{2}+\sigma_{d}^{j^{2}}}
$$

where;

$\boldsymbol{g}_{k}^{j}=$ complex signal of $f^{\text {th }}$ destination antennas where, $j=1, \cdots, N_{d}$

$\sigma_{d}^{j^{2}}=$ noise variance at the destination i.e. $\sigma_{d}^{j^{2}}=\sigma_{1}^{j^{2}}, \cdots, \sigma_{d}^{j^{2}}$

$\boldsymbol{f}_{k}^{j}=$ vector-by-vector $k^{\text {th }}$ RTP for $f^{\text {th }}$ antenna elements i.e. $f_{k}^{j}=f_{1}, \cdots, f_{N_{d}}$ When (15) is maximized, the precoding vectors per antenna are generated as

$$
\begin{gathered}
\boldsymbol{f}_{k}^{j}=\arg \max _{\boldsymbol{F}_{k}^{j} \in C^{N_{r} \times 1}} \frac{\boldsymbol{f}_{k}^{j^{H}}\left(\boldsymbol{g}_{k}^{j^{H}} \boldsymbol{g}_{k}^{j}\right) \boldsymbol{f}_{k}^{j}}{\boldsymbol{f}_{k}^{j^{H}}\left(\boldsymbol{G}_{k}^{j^{H}} \boldsymbol{G}_{k}^{j}+\sigma_{r k}^{j^{2}} \boldsymbol{I}_{N_{r}}\right) \boldsymbol{f}_{k}^{j}} \\
\boldsymbol{F}_{k}^{j}=\left[\boldsymbol{f}^{1}, \cdots, \boldsymbol{f}^{N_{d}}\right]
\end{gathered}
$$

Here “ $\boldsymbol{F}_{k}^{j}$ ” represents the $k^{\text {th }}$ RTP matrix for $f^{\text {th }}$ receive antennas at the destination, which supports maximizing the SLNR.

For this objective function, different precoders are selected to maximize the effectiveness of the wanted signal analogous to the interference and noise due to the leakage. The multiplication of relay receive beamforming matrix from (11) and (16) gives:

$$
\boldsymbol{F}=\boldsymbol{F}_{k}^{j} \times \boldsymbol{B}_{k} .
$$

\subsection{Fukunaga-Koontz Transform (FKT) Based Relay Receive Beamforming Algorithm}

For the selection of feature, Koontz and Fukunaga suggested a statistical method for high-dimensional data [23]. It was meant for a two-class reorganization problem i.e. set of vectors that represent two classes, concurrently the vectors that best represent one class will be the least representative one for the other class [28]. Let $\boldsymbol{Z}_{1}$ and $\boldsymbol{Z}_{2}$ be two data matrices, then $\boldsymbol{S}_{1}=\boldsymbol{Z}_{1} \boldsymbol{Z}_{1}^{\mathrm{T}}$ and $\boldsymbol{S}_{2}=\boldsymbol{Z}_{2} \boldsymbol{Z}_{2}^{\mathrm{T}}$ will be the symmetric auto correlation and positive semi definite matrices. The covariance of the two matrices $\boldsymbol{S}_{1}$ and $\boldsymbol{S}_{2}$ be computed essentially, according to the FKT. The subsequent phase of the algorithm is to add up $\boldsymbol{S}_{1}$ and $\boldsymbol{S}_{2}$ as $\boldsymbol{S}=\boldsymbol{Z}_{1} \boldsymbol{Z}_{1}^{\mathrm{T}}+\boldsymbol{Z}_{2} \boldsymbol{Z}_{2}^{\mathrm{T}}$ and decomposing the matrix $\boldsymbol{S}$ into eigenvalues and eigenvectors by using singular value decomposition i.e. $\boldsymbol{S}=\left[\boldsymbol{U D} \boldsymbol{V}^{\mathrm{T}}\right]$. 
The $\boldsymbol{U}$ and $\boldsymbol{D}$ matrices are used to build the transformation operator $\boldsymbol{P}$, which is also termed as the FKT [29]. It is used to alter the data into the eigenspace and is given by:

$$
\boldsymbol{P}=\boldsymbol{U} \boldsymbol{D}^{-1 / 2}
$$

$\boldsymbol{D}=$ diagonal matrix with eigenvalues, which are non-zero and positive singular values i.e. $\boldsymbol{D}=\operatorname{diag}\left\{\lambda_{1}, \cdots, \lambda_{r}\right\}, \quad \lambda_{1} \geq \cdots \geq \lambda_{r}>0$.

$\boldsymbol{U}=$ identity matrix.

The matrices $\boldsymbol{S}_{1}$ and $\boldsymbol{S}_{2}$ are transformed into subspace by using the $\boldsymbol{P}$ operator with lesser dimension. After the transformation, two new matrices i.e. $\tilde{\boldsymbol{S}}_{1}$ and $\tilde{\boldsymbol{S}}_{2}$ are obtained which are given as:

$$
\tilde{\boldsymbol{S}}_{1}=\boldsymbol{P}^{\mathrm{T}} \boldsymbol{S P} \text { and } \tilde{\boldsymbol{S}}_{2}=\boldsymbol{P}^{\mathrm{T}} \boldsymbol{S P}
$$

where $\tilde{\boldsymbol{S}}_{1}$ and $\tilde{\boldsymbol{S}}_{2}$ are the transformed target and background matrices and their sum should be equal to the identity matrix i.e.

$$
\tilde{\boldsymbol{S}}_{1}+\tilde{\boldsymbol{S}}_{2}=\boldsymbol{I} \text {, and } \tilde{\boldsymbol{S}}_{1}=\boldsymbol{I}-\tilde{\boldsymbol{S}}_{2} .
$$

The same eigenvectors and eigenvalues are shared by $\tilde{\boldsymbol{S}}_{1}$ and $\tilde{\boldsymbol{S}}_{2}$ as these are transformed by using the same operator. If $\boldsymbol{v}$ is the eigenvector of $\tilde{\boldsymbol{S}}_{1}$ with the eigenvalue $\lambda_{1}$ then, $\tilde{S}_{1} v=\lambda_{1} v$ [30].

$$
\begin{aligned}
& \left(\boldsymbol{I}-\tilde{\boldsymbol{S}}_{2}\right) \boldsymbol{v}=\lambda_{1} \boldsymbol{v} . \\
& \tilde{\boldsymbol{S}}_{2} \boldsymbol{v}=\left(1-\lambda_{1}\right) \boldsymbol{v} .
\end{aligned}
$$

In (18), $\tilde{\boldsymbol{S}}_{1}$ holds important information for the targeted class whereas, $\tilde{\boldsymbol{S}}_{2}$ has more significant information for the background class. The training stage is completed after obtaining these two matrices.

The optimization problem of the relay receive beamforming for $k$ number of relays is formulated to FKT as follows:

$$
\begin{gathered}
\tilde{\boldsymbol{S}}_{1}=\boldsymbol{h}_{k}^{\boldsymbol{l}^{H}} \boldsymbol{h}_{k}^{l} . \\
\tilde{\boldsymbol{S}}_{2}=\boldsymbol{H}_{k}^{H} \boldsymbol{H}_{k}+\sigma_{r k}^{2} \boldsymbol{I}_{N_{r}} .
\end{gathered}
$$

And their sum is given as:

$$
\boldsymbol{S}=\tilde{\boldsymbol{S}}_{1}+\tilde{\boldsymbol{S}}_{2}=\boldsymbol{H}_{c}^{H} \boldsymbol{H}_{c}+\sigma_{r k}^{2} \boldsymbol{I}_{N_{r}} .
$$

The FKT factor can be calculated by using the facts of (21) and (22) for individual relay receive antenna, which generates the shared eigenspace matrices $\tilde{\boldsymbol{S}}_{1}$ and $\tilde{\boldsymbol{S}}_{2}$. They complement one another in a manner that the best-principled eigenvectors of the matrix $\tilde{S}_{1}$ are the least principled eigenvectors for the matrix $\tilde{\boldsymbol{S}}_{2}$ and vice versa. The FKT factor, after multiplying with the eigenvectors of unitary matrix $\boldsymbol{U}$, gives the eigenvalue of matrix $\boldsymbol{D}$ of transformed antenna covariance matrix [24] [31]. It is important to note that, for a cluster of relay nodes, the computation of FKT is required only one time that reduces the computational load.

The summary of the algorithm steps to compute the FKT factor for MR nodes 
is given in Table 1. Starting from step one to step two the FKT is computed, in step three and four beamforming is generated for the $k^{\text {th }}$ number of relays.

The received signal is given as:

$$
y_{d}=\sum_{\substack{j=1 \\ k \neq j}}^{N_{d}} \rho \boldsymbol{G F H s}+\sum_{\substack{j=1 \\ k \neq j}}^{N_{d}} \boldsymbol{G} \boldsymbol{F} \boldsymbol{s}_{i}+\boldsymbol{G} \boldsymbol{F} \boldsymbol{n}_{r k}+\boldsymbol{n}_{d}
$$

where;

$$
\begin{aligned}
& \boldsymbol{G}_{s d}=\rho \boldsymbol{G} \boldsymbol{F H} \\
& \boldsymbol{I}=\boldsymbol{G} \boldsymbol{F} \boldsymbol{s}_{i} \quad \text { (Interference due to signal leakage) } \\
& \mathbf{z}=\boldsymbol{G} \boldsymbol{F} \boldsymbol{n}_{r k}+\boldsymbol{n}_{d} \quad \text { (Noise at the destination). }
\end{aligned}
$$

According to [14], the filter used at the destination is based on QRD. The QRD operation of the equivalent channel $\boldsymbol{G}_{s d}$ provides two output matrices $\boldsymbol{Q}$ and $\boldsymbol{R}$, which are the $m \times n$ unitary and upper triangular matrices respectively. It is given as

$$
\boldsymbol{R}=\left[\begin{array}{ccc}
r_{11} & r_{12} & r_{13} \\
0 & r_{22} & r_{23} \\
0 & 0 & r_{33}
\end{array}\right] .
$$

The destination filter based on QRD is given as:

$$
\boldsymbol{W}=\left(\boldsymbol{Q}_{s d}\right)^{H} .
$$

The SINR is obtained when the transmitted signal is divided by the interfering signals and noise [32]. The SINR can be expressed as follows:

$$
\operatorname{SINR}=\frac{\left(\frac{P_{s}}{N_{d}}\right) r_{j, j}^{2}}{\left\|\left(\rho\left(\boldsymbol{Q}_{s d}\right)^{H} \boldsymbol{G} \boldsymbol{F}\right)_{j}\right\|^{2} \sigma_{r}^{l^{2}}+\sigma_{d}^{j^{2}}} .
$$

where $r_{j, j}^{2}$ are $f^{\text {th }}$ entries in the diagonal of the matrix “ $\boldsymbol{R}$ ”. Finally, the network ergodic capacity is given as:

Table 1. Porcessing algorithm.

\begin{tabular}{ll}
\hline Steps & Activities \\
\hline Input & SR channel matrices and noise variance at the relay terminal $\left(\boldsymbol{H}_{k}\right.$ and $\left.\sigma_{r}^{l^{2}}\right)$ \\
1. & Calculation ofthe summation of covariance matrices $\tilde{\boldsymbol{S}}_{1}$ and $\tilde{\boldsymbol{S}}_{2}$. \\
2. & Computation of the transform factor $\boldsymbol{P}$ from singular value decomposition of $\boldsymbol{S}$ \\
3. & For $l=1, \cdots, N_{k}$ relay receive antennas \\
4. & For $k=1, \cdots, K$ number of relays \\
& Using the FKT factor, transform the $I^{\mathrm{h}}$ receive antennas covariance matrix $\tilde{\boldsymbol{S}}_{1}$ from $(21)$ \\
& Transformation of $\boldsymbol{P}$ to $\tilde{\boldsymbol{S}}_{1}$ and selection of the first eigenvector $\boldsymbol{v}^{l}$ of matrix $\tilde{\boldsymbol{S}}_{1}$ \\
& $\boldsymbol{b}_{k}^{l}=\boldsymbol{P}^{l}$ is the relay receive beamforming vector for $t^{\mathrm{h}}$ antennasand $k$ relay receive \\
antennas & Multiplication of $\boldsymbol{B}_{k}^{l}$ with $\boldsymbol{F}^{j}$ \\
5. & End \\
Output & $\boldsymbol{F}=\boldsymbol{B}_{k}^{l} \times \boldsymbol{F}^{j}$ the relay precoding matrix \\
\end{tabular}




$$
C_{\text {erg }}=E_{\{H, G\}}\left\{\frac{1}{2} \sum_{\substack{j=1 \\ k \neq j}}^{N_{d}} \log _{2}(1+\operatorname{SINR})\right\}
$$

For the dual-hop multi-relay assisted network, the upper-bound capacity is given as:

$$
C_{\text {upper }}=E_{\left\{H_{k}\right\}_{k=1}^{K}}\left\{\frac{1}{2} \log _{2} \operatorname{det}\left(\boldsymbol{I}_{N_{s}}+\frac{P_{s}}{N_{s} \sigma_{r}^{2}} \sum_{k=1}^{K} \boldsymbol{H}^{H} \boldsymbol{H}\right)\right\}
$$

where,

$P_{s}=$ total source transmit power.

$1 / 2$ = time-sharing factor (for signals of the receiving and forwarding process.

\section{Simulation Methodology}

In this section, the MCS is used to present the theoretical results of the proposed scheme. In all simulations, dimensionally uncorrelated MIMO channel matrices are developed between the source to the relay nodes and the relay nodes to the destination without loss of generality. These matrices are identically distributed, independent, and their entries are CG random variables with zero-mean components and unit-variance i.e. $C N(0,1)$. At the relay and destination terminal, the noise variances are considered equal i.e.

$$
\sigma_{r}^{2}=\sigma_{d}^{2}
$$

For the validation of the performance of the proposed scheme, the performance results of the existing techniques are compared.

The performance of a DH-MR-MIMO network is evaluated in terms of the ergodic capacity. From the source node, independent data streams are transmitted by using separate antennas having equal distribution power. The SNR of the S-R and R-D channels are given as $S N R_{s r}=P_{s} / \sigma_{r}^{2}$ and $S N R_{r d}=P_{k} / \sigma_{d}^{2}$. The channel knowledge is considered at the relay terminals for S-R and R-D channels [14]. For the DH-MR-MIMO network, the upper-bound capacity is considered as a reference point [11].

The results from the MCS of the recommended beamforming technique for the DH-MR-network are balanced for 5000 independent channels to evaluate the performance of the suggested scheme. The performance of other available precoding methods that are discussed in [23], as well as ZF-ZF, MF-RZF, and MFZF techniques are evaluated that are explained in [13] [14] and [17] respectively. Table 2 represents the parameters used in the simulation process.

\section{Result and Discussion}

This section discusses the evaluation of the suggested and compared schemes. The results are obtained by using different number of antennas at each node by taking various number of relay nodes. The ergodic capacity curves of the proposed scheme and other beamforming techniques are compared and shown in Figure 2. The results are obtained with $N_{s}=N_{r}=N_{d}=3$ at each node and one relay between the source and destination. 
The graph shows the results of the suggested and additional beamforming techniques. It is clear from the figure that at each SNR value, the recommended technique outperforms other techniques.

The regularization factor is used for the suggested scheme, which clearly shows that the MF-ZF technique is outperformed by the MF-RZF technique. When the value of the regularization parameter is reduced, then the scheme approaches the MF-ZF beamforming. Since the MF technique performs well at low SNR values, the MF-ZF technique outperforms the ZF-ZF technique only when the SNR values are low, although the interference is eliminated by the ZF beamforming that will cost the noise enhancement. The results clearly show that at low SNR values, the ZF-ZF scheme gives slow performance but it regains its performance at a high $\mathrm{SNR}$ value.

The plots of Figures 3-5 represent the efficiency of the suggested beamforming scheme at an increased number of relay nodes from one to six by keeping the

Table 2. Parameters for simulation.

\begin{tabular}{cc}
\hline Parameters & Value \\
\hline InputRelay protocol & Amplify-and-forward \\
Network configuration & DH-MR-MIMO system as shown in Figure 1 \\
Antenna configurations at each node & $N_{s}=N_{r}=N_{d}=3$, and $N_{s}=N_{r}=N_{d}=6$ \\
Noise variance & $\sigma_{r}^{2}=\sigma_{d}^{2}$ \\
Channel between S-R and R-D is assumed & Rayleigh flat fading \\
$S N R_{s r}$ & $S N R_{s r}=P_{s} / \sigma_{r}^{2}$ \\
$S N R_{r d}$ & $S N R_{r d}=P_{k} / \sigma_{d}^{2}$ \\
Noise & AWGN \\
CSI & Considered perfect at the relay terminals \\
\hline
\end{tabular}

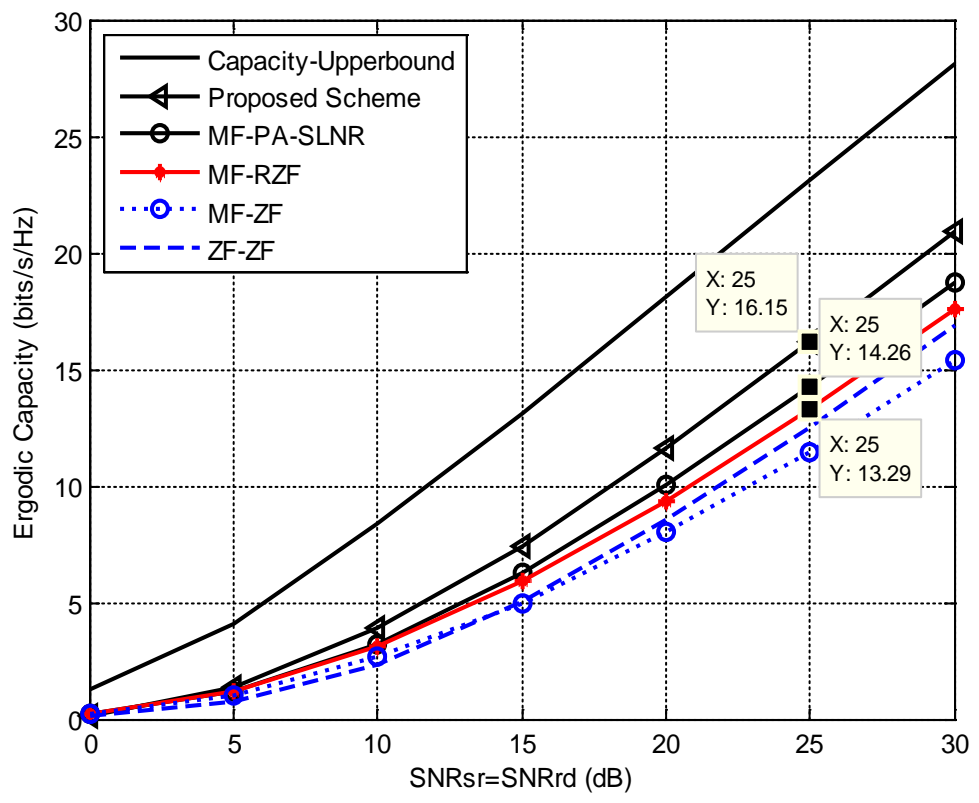

Figure 2. Maximum ergodic capacity (bits/s/Hz) v/s SNR. 
same number of antennas i.e. three antennas at each node of the network. The distributed array gain is achieved from MR nodes, which helps in increasing the ergodic capacity, whereas, diversity gain is achieved by the number of antennas at each node. The efficiency of the proposed technique is validated by its performance gain against the interference in contrast with other techniques that control the interference between several antennas of MR nodes and destination nodes.

The results achieved by the suggested technique and other beamforming techniques are represented in the graph. The network is equipped with

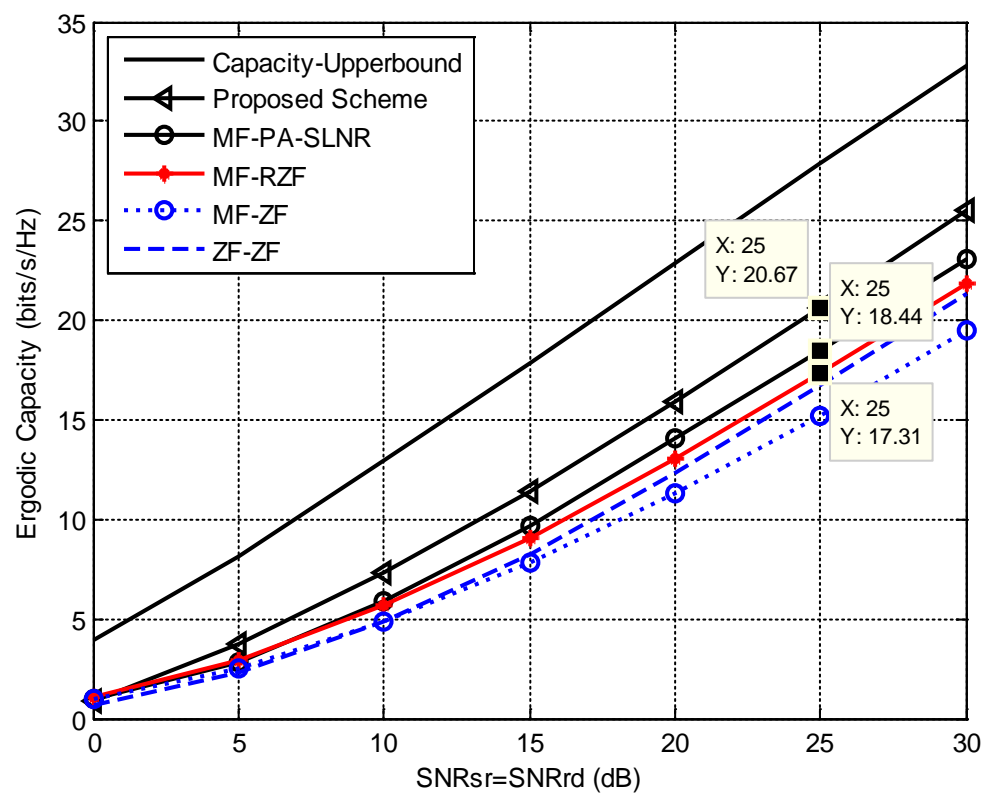

Figure 3. Maximum ergodic capacity (bits/s/Hz) v/s SNR.

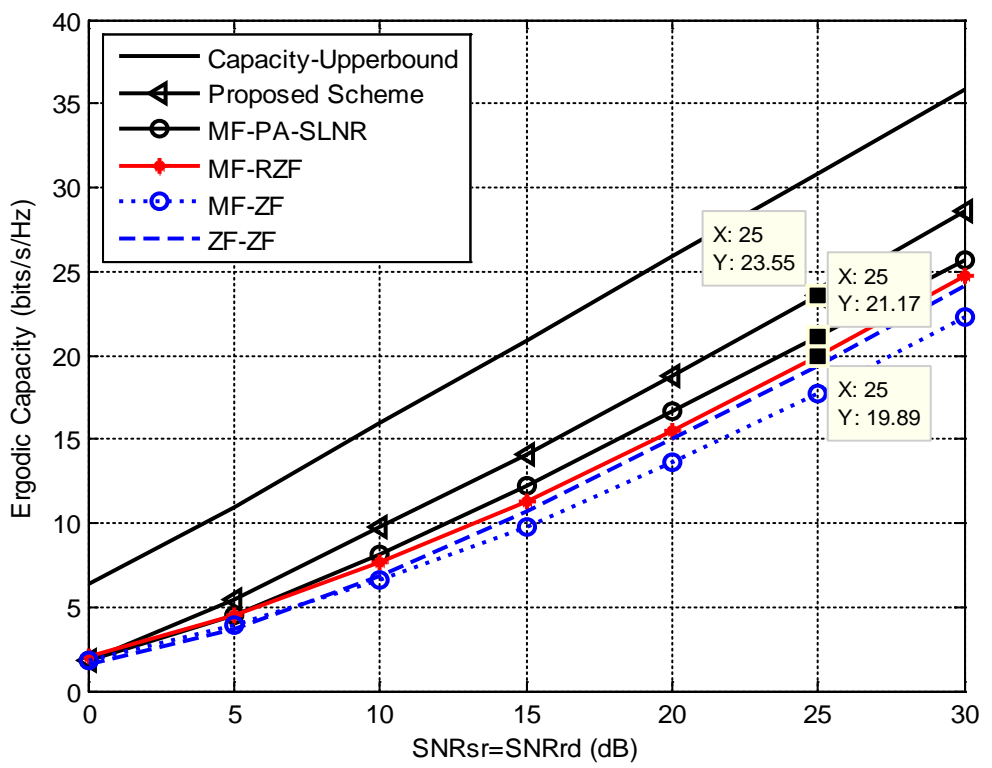

Figure 4. Maximum ergodic capacity (bits/s/Hz) v/s SNR. 


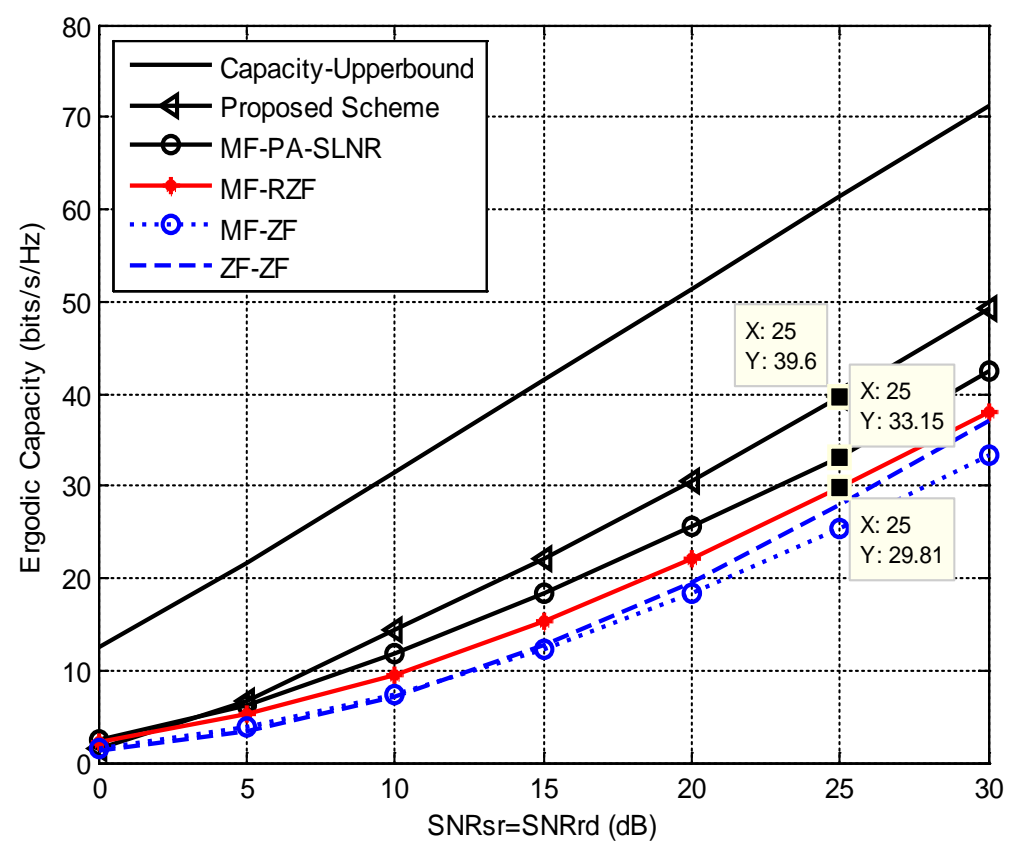

Figure 5. Network capacity in bits/s/Hz v/s SNR.

$N_{s}=N_{r}=N_{d}=3$ and $K=3$ ( $K$ is the number of relay nodes with three antennas at each node).

The results achieved by the suggested technique and other beamforming techniques are represented in the graph. The network is equipped with $N_{s}=N_{r}=N_{d}=3$ and $K=6$.

The graph represents the performance comparisons of the suggested scheme with other precoding schemes having perfect CSI at the relay node, under system configuration of $N_{s}=N_{r}=N_{d}=6$ and $K=6$.

Table 3 represents the capacity gained at $25 \mathrm{~dB}$ SNR by suggested and other techniques by keeping three antennas at each node and repeating the simulation process for various relay nodes such as 1,3 , and 6 . The performance of the proposed beamforming technique is much improved as compared to the other techniques when applied to the MR-MIMO network. There is a linear increase in the capacity as the number of relay nodes increases.

Figure 6 shows the response of the proposed technology in terms of ergodic capacity and SNR. It represents the linear behavior of the proposed relay precoding technique at $N_{s}=N_{r}=N_{d}=3$. The simulation results are obtained with a large number of relay nodes and SNR values. It is observed that the proposed technique gives improved outcomes that can reach the upper-bound capacity limit.

Figure 7 compares the plots of the ergodic capacity of the suggested technique for several relay nodes. It shows that for fixed number of antennas, as the number of relay nodes escalates, the ergodic capacity of the network increases.

The simulation results show that when SINR-Max is considered for beamforming, the design gives better performance alongside the inter-stream interference at 
Table 3. Parameters for simulation.

\begin{tabular}{|c|c|c|c|c|}
\hline \multirow{4}{*}{ No. } & \multirow{4}{*}{$\begin{array}{l}\text { Beamforming at } \\
\text { the AF relay nodes }\end{array}$} & \multicolumn{3}{|c|}{$\begin{array}{l}\text { Capacity gain for } 3 \text { antennas at each node } \\
\text { and different number of relay nodes }\end{array}$} \\
\hline & & \multicolumn{3}{|c|}{ Number of relay nodes } \\
\hline & & 1 & 2 & 3 \\
\hline & & $\mathrm{bit} / \mathrm{s} / \mathrm{Hz}$ & $\mathrm{bit} / \mathrm{s} / \mathrm{Hz}$ & $\mathrm{bit} / \mathrm{s} / \mathrm{Hz}$ \\
\hline 1 & Proposed technique & 16.15 & 20.67 & 23.55 \\
\hline 2 & MF-PA-SLNR & 14.26 & 18.44 & 21.17 \\
\hline 3 & MF-RZF & 13.26 & 17.31 & 19.80 \\
\hline
\end{tabular}

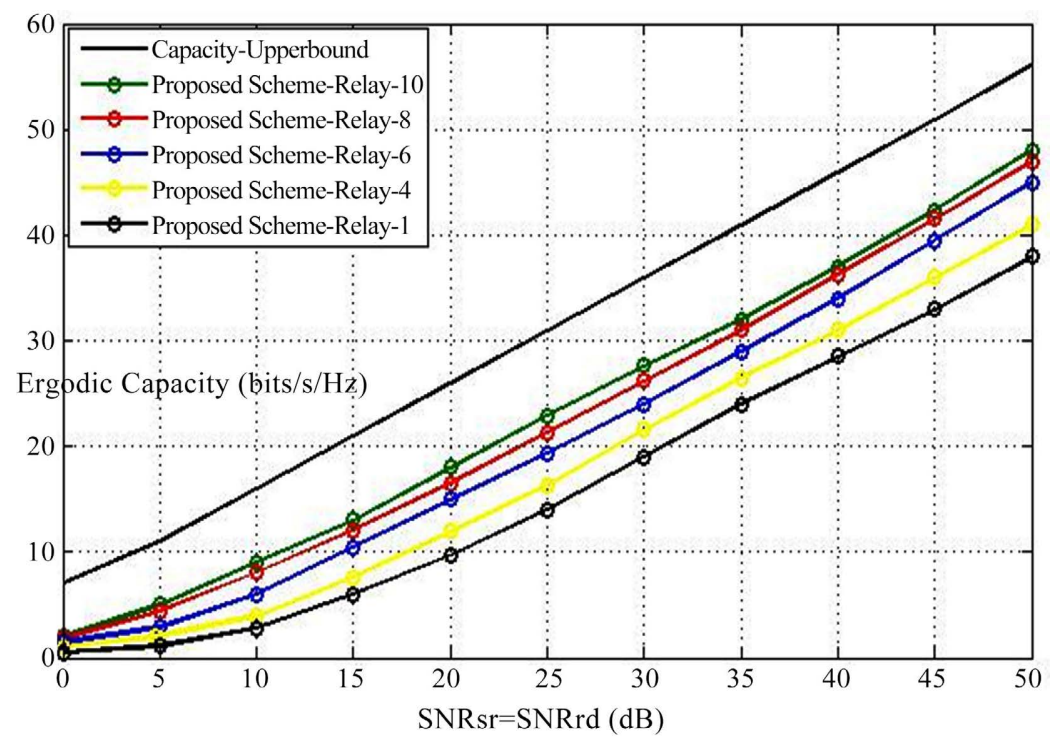

Figure 6. Network ergodic capacity (bits/s/Hz) v/s SNR (dB).

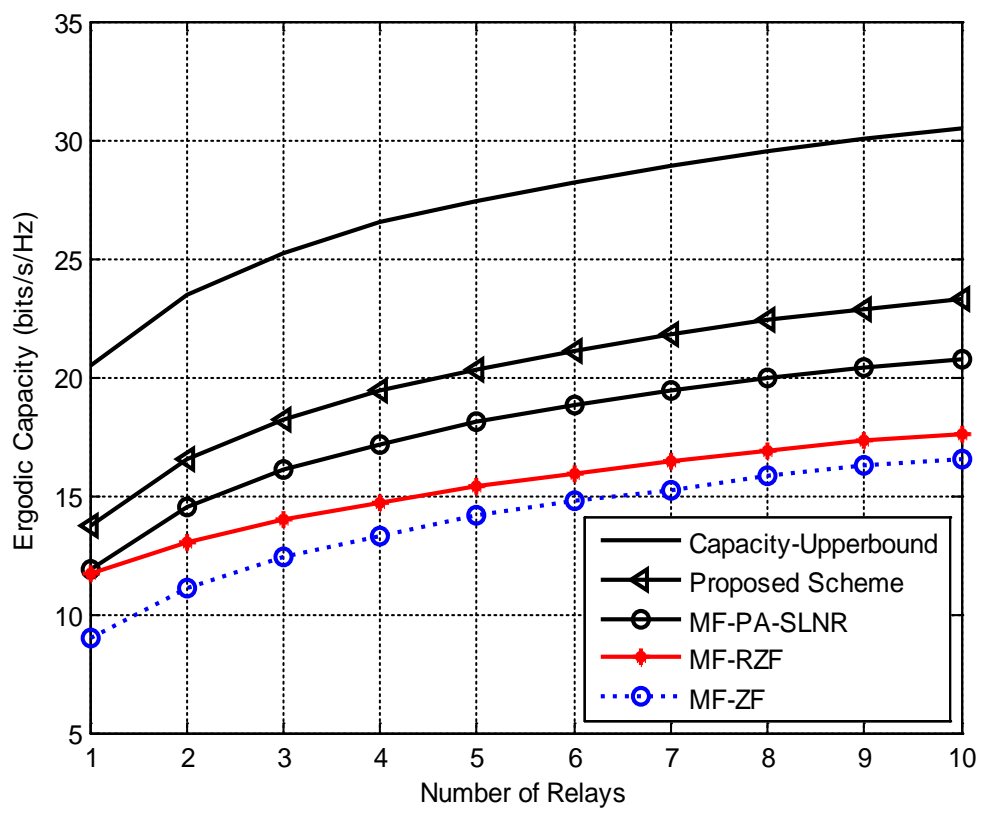

Figure 7. Maximum ergodic capacity v/s number of AF relays. 
MR nodes. Whereas, the relay downlink provides unwanted signal reduction due to the signal leakage at the destination. Moreover, when the FKT is used in the proposed technique, it effectively solves the issue of optimization. It is clear from the FKT algorithm that the computational burden of beamforming is reduced because the transformation factor needs to be computed only one time for MRs.

However, when the number of antennas is increased at each terminal from three to four, the measured sum capacity using the proposed scheme is increased as compared to the MF beamforming at the relay receive side. Similarly, as the number of antennas rises to eight, the sum capacity also increases linearly. Based on the results and discussions mentioned above, it is concluded that the proposed technique for AF relay is more efficient as compared to other techniques. The performance of the whole system is enhanced in terms of the ergodic capacity by adding more relays due to the diversity order achieved by an additional number of relays. Moreover, the increasing number of antennas at individual nodes increases the capacity and decreases the number of relay nodes, which may result in complexity reduction.

\section{Conclusions and Future Direction}

A linear beamforming scheme has been proposed using the Fukunaga-Koontz Transform (FKT) to control the interference for amplify and forward multiple relay MIMO assisted dual-hop network, where the co-channel interference (CCI) was considered at relay receivers and interference at the destination node due to leakage signal. The performance of the proposed scheme demonstrates that by considering interference mitigation criteria the ergodic capacity at a significant level is improved as compared to the conventional techniques. Therefore, the proposed techniques based on the maximization of the signal-to-interference-plus-noise ratio (SINR) and signal-to-leakage-and-noise ratio (SLNR) relay processing outperform other conventional techniques in terms of ergodic capacity.

The results in this work provide a strong foundation for future work in the performance of wireless network. One area of the future work is to optimize the Performance of Massive MIMO systems, to limit inter-cell interference and to improve the use of the available frequency bands by covering cognitive radio and mobile to mobile communications(D2D), to guarantee a level of end-to-end quality of service in different mobile wireless environments. Another area is maturing enough beamforming algorithms in order to exploit Millimeter wave to its full potential. It requires improvement in the networks when personal base stations (BSs) and satellite connectivity can get merged into cellular communication.

\section{Acknowledgements}

This work would not have been possible without the financial support of Doctoral School of Science and Technology of the University of N'Djamena's, EcoleNationaleSuperieure des Technologies de l'Informationet de la Communication (ENASTIC) and Islamic Developement Bank (IsDB). 


\section{Conflicts of Interest}

The authors declare no conflicts of interest regarding the publication of this paper.

\section{References}

[1] Dohler, M. and Li, Y. (2010) Cooperative Communications: Hardware, Channel and PHY. John Wiley \& Sons, Hoboken.

[2] Van Der Meulen, E.C. (1971) Three-Terminal Communication Channels. Advances in Applied Probability, 3, 120-154. https://doi.org/10.2307/1426331

[3] Zhioua, G.E.M., Labiod, H., Tabbane, N. and Tabbane, S. (2013) LTE Advanced Relaying Standard: A Survey. Wireless Personal Communications, 72, 2445-2463. https://doi.org/10.1007/s11277-013-1157-1

[4] Chandra, A., Bose, C. and Bose, M.K. (2011) Wireless Relays for Next Generation Broadband Networks. IEEE Potentials, 30, 39-43.

https://doi.org/10.1109/MPOT.2011.940778

[5] Berger, S., Kuhn, M., Wittneben, A., Unger, T. and Klein, A. (2009) Recent Advances in Amplify-and-Forward Two-Hop Relaying. IEEE Communications Magazine, 47, 50-56. https://doi.org/10.1109/MCOM.2009.5183472

[6] Zhou, P., et al. (2018) IEEE 802.11ay-Based mmWave WLANs: Design Challenges and Solutions. IEEE Communications Surveys and Tutorials, 20, 1654-1681. https://doi.org/10.1109/COMST.2018.2816920

[7] Roh, W., et al. (2014) Millimeter-Wave Beamforming as an Enabling Technology for 5G Cellular Communications: Theoretical Feasibility and Prototype Results. IEEE Communications Magazine, 52, 106-113. https://doi.org/10.1109/MCOM.2014.6736750

[8] Zhou, P., et al. (2017) Enhanced Random Access and Beam Training for Millimeter Wave Wireless Local Networks with High User Density. IEEE Transactions on Wireless Communications, 16, 7760-7773. https://doi.org/10.1109/TWC.2017.2753779

[9] Huang, M., Chi, T., Wang, F. and Wang, H. (2017) An All-Passive Negative Feedback Network for Broadband and Wide Field-of-View Self-Steering Beam-Forming with Zero DC Power Consumption. IEEE Journal of Solid-State Circuits, 52, 1260-1273. https://doi.org/10.1109/JSSC.2016.2641947

[10] Bolt, M. and Adams, M. (2020) A High-Speed DLL-Based Hybrid Phase Conjugator for 5G Beamforming. Circuits and Systems, 11, 27-38.

https://doi.org/10.4236/cs.2020.113003

[11] Bolcskei, H., Nabar, R.U., Oyman, O. and Paulraj, A.J. (2006) Capacity Scaling Laws in MIMO Relay Networks. IEEE Transactions on Wireless Communications, 5, 1433 1444. https://doi.org/10.1109/TWC.2006.1638664

[12] Ding, Y., Zhang, J.K. and Wong, K.M. (2009) Ergodic Channel Capacities for the Amplify-and-Forward Half-Duplex Cooperative Systems. IEEE Transactions on Information Theory, 55, 713-730. https://doi.org/10.1109/TIT.2008.2009822

[13] Shi, H., Abe, T., Asai, T. and Yoshino, H. (2007) Relaying Schemes Using Matrix Triangularization for MIMO Wireless Networks. IEEE Transactions on Communications, 55, 1683-1688. https://doi.org/10.1109/TCOMM.2007.904356

[14] Zhang, Y., Luo, H. and Chen, W. (2010) Efficient Relay Beamforming Design with SIC Detection for Dual-Hop MIMO Relay Networks. IEEE Transactions on Vehicular Technology, 59, 4192-4197. https://doi.org/10.1109/TVT.2010.2065249 
[15] Wang, Z. and Chen, W. (2013) Relay Beamforming Design with SIC Detection for MIMO Multi-Relay Networks with Imperfect CSI. IEEE Transactions on Vehicular Technology, 62, 3774-3785.

[16] Wang, Z., Chen, W. and Li, J. (2012) Efficient Beamforming for MIMO Relaying Broadcast Channel with Imperfect Channel Estimation. IEEE Transactions on Vehicular Technology, 61, 419-426. https://doi.org/10.1109/TVT.2011.2173590

[17] Wang, Z., Chen, W., Gao, F. and Li, J. (2011) Capacity Performance of Relay Beamformings for MIMO Multirelay Networks with Imperfect $R$ - $D$ CSI at Relays. IEEE Transactions on Vehicular Technology, 60, 2608-2619. https://doi.org/10.1109/TVT.2011.2157546

[18] Senaratne, D. and Tellambura, C. (2009) Performance Analysis of Channel Inversion over MIMO Channels. GLOBECOM 2009-2009 IEEE Global Telecommunications Conference, Honolulu, 30 November-4 December 2009, 1-5. https://doi.org/10.1109/GLOCOM.2009.5425727

[19] Peel, C., Spencer, Q., Swindlehurst, A.L. and Hochwald, B. (2004) Downlink Transmit Beamforming in Multi-User MIMO Systems. Sensor Array and Multichannel Signal Processing Workshop Proceedings, 43-51.

[20] Jing, J. and Zheng, X. (2014) A Downlink Max-SINR Precoding for Massive MIMO System. International Journal of Future Generation Communication \& Networking, 7, 107-116. https://doi.org/10.14257/ijfgcn.2014.7.3.10

[21] Huang, Y., Li, C., Zhong, C., Wang, J., Cheng, Y. and Wu, Q. (2013) On the Capacity of Dual-Hop Multiple Antenna AF Relaying Systems with Feedback Delay and CCI. IEEE Communications Letters, IEEE, 17, 1200-1203. https://doi.org/10.1109/LCOMM.2013.051313.130560

[22] Saand, A.S., Jeoti, V., Saad, M. and Naufal, M. (2014) Relay Precoding for Dual-Hop MIMO Network Using per Antenna SLNR Maximization. 2014 IEEE Symposium on Wireless Technology and Applications (ISWTA), Kota Kinabalu, 28 September-1 October 2004, 41-46. https://doi.org/10.1109/ISWTA.2014.6981192

[23] Fukunaga, K. and Koontz, W.L. (1970) Application of the Karhunen-Loève Expansion to Feature Selection and Ordering. IEEE Transactions on Computers, 19, 311 318. https://doi.org/10.1109/T-C.1970.222918

[24] Saeid, E., Jeoti, V. and Samir, B.B. (2012) Efficient PA-SLNR Linear Precoding for Multiuser Multiple Input Multiple Output System. Research Journal of Applied Sciences, Engineering and Technology, 4, 2489-2495.

[25] Huang, C. and Zhang, X.P. (2013) Performances of Amplify-and-Forward Cooperative Relay Networks with Different Topologies. Wireless Personal Communications, 69, 561-577. https://doi.org/10.1007/s11277-012-0590-x

[26] Lee, K.J., Sung, H., Park, E. and Lee, I. (2010) Joint Optimization for One and TwoWay MIMO AF Multiple-Relay Systems. IEEE Transactions on Wireless Communications, 9, 3671-3681. https://doi.org/10.1109/TWC.2010.102210.091021

[27] Sibille, A., Oestges, C. and Zanella, A. (2010) MIMO: From Theory to Implementation. Academic Press, Cambridge, MA.

[28] Zheng, W. and Lin, Z. (2012) A New Discriminant Subspace Analysis Approach for Multi-Class Problems. Pattern Recognition, 45, 1426-1435. https://doi.org/10.1016/j.patcog.2011.10.021

[29] Dinc, S. and Bal, A. (2011) A Statistical Approach for Multiclass Target Detection. Procedia Computer Science, 6, 225-230. https://doi.org/10.1016/j.procs.2011.08.042

[30] Zhang, S. and Sim, T. (2006) When Fisher Meets Fukunaga-Koontz: A New Look at Linear Discriminants. 2006 IEEE Computer Society Conference on Computer Vision 
and Pattern Recognition, New York, 17-22 June 2006, 323-329.

https://doi.org/10.1109/CVPR.2006.334

[31] Saeid, E., Jeoti, V. and Samir, B.B. (2013) On MU-MIMO Precoding Techniques for WiMAX. https://doi.org/10.5772/56034

[32] Adam, E.E., Li, Y., Haruna, R. and Mohammed, A.A. (2014) Performance Analysis of Best Relaying Protocol Selection with Interferences at Relays. Radioengineering, 23, 570-577. 


\section{Abbreviations}

\begin{tabular}{cc}
\hline Abbreviations & Description \\
\hline AF & Amplify-and-forward \\
AWGN & Additive White Gaussian Noise \\
CSI & Channel state information \\
CN $(0,1)$ & Complex circular symmetric Gaussian distribution \\
DF & Decode and forward \\
i.i.d & Independent and identically distributed \\
LTE & Long term evolution \\
FKT & Fukunaga-Koontz Transform \\
S-R & Source to Relay \\
RZF & Regularized zero-forcing \\
R-D & Relay to Destination \\
SLNR & Signal-to-leakage-and-noise ratio \\
SINR & Signal-to-interference-plus-noise ratio \\
ZF & Zero-forcing \\
\hline
\end{tabular}

\section{Notations}

\begin{tabular}{cc}
\hline Notations & Description \\
\hline Lowercase boldface letters & Vectors \\
Uppercase boldface letter & Matrices \\
$t r()$. & Trace function \\
$C N(0,1)$ & Complex circular symmetric Gaussian distribution \\
$(.)^{H}$ & Hermitian transpose \\
$|\cdot|$ & Determinant \\
$\|s\|$ & Euclidean norm of a vector $s$ \\
$\|\boldsymbol{X}\|_{F}$ & Frobenius norm matrix $\boldsymbol{X}$ \\
$\boldsymbol{I}_{N_{s}}$ & Identity matrix of $N_{s} \times N_{s}$ dimension \\
$E()$. & Expectation operation \\
$\boldsymbol{H}_{k}$ & Channel matrix between source to $k$ relay nodes \\
$\boldsymbol{H}^{\mathrm{T}}$ & Transpose of matrix $\boldsymbol{H}$ \\
$\boldsymbol{H}^{H}$ & Hermitian of matrix $\boldsymbol{H}$ \\
$\log _{2}$ & The logarithm with base 2 \\
$\sigma_{r}^{2}$ & The noise variance at relay node \\
$\sigma_{d}^{2}$ &
\end{tabular}

